\title{
AN INDEX THEOREM FOR $p$-ADIC DIFFERENTIAL OPERATORS
}

\author{
BY
}

\author{
A. ADOLPHSON( $\left.{ }^{1}\right)$
}

\begin{abstract}
A system of first order linear differential operators satisfying conditions arising naturally in geometry (rational function coefficients, regular singularities, non-Liouville exponents) is considered. It is shown that the index of the system on certain spaces of holomorphic functions can be calculated by restricting to a subspace of rational functions. This is applied to obtain an explicit formula for the index of a single $k$ th order linear differential operator.
\end{abstract}

1. Introduction. Let $p$ be a prime and let $\mathrm{Q}_{p}$ denote the $p$-adic rationals. Let $\mathbf{C}_{p}$ be the completion of the algebraic closure of $\mathbf{Q}_{p}$. Then $\mathbf{C}_{p}$ is algebraically closed and has a (nondiscrete) additive valuation "ord", which we suppose normalized so that ord $p=1$. The object of this work is to calculate the index of certain types of differential operators acting on spaces of $p$-adic holomorphic functions, i.e., functions holomorphic on certain subsets of $\mathbf{C}_{p}$. Our main result is Theorem 2, which says that under certain restrictions the index of a differential operator is the same whether computed on holomorphic functions or on rational functions whose poles are required to lie in a certain finite set. The value of such a result lies in the fact that it is usually easy to explicitly calculate the index on rational functions (see $\S 4$, especially Proposition 1 and its corollaries).

We will show that the holomorphic and rational kernels (respectively cokernels) are the same (respectively: isomorphic). The identity of the kernels (Lemma 3) will follow trivially from our hypotheses. To show the cokernels are isomorphic, we first show they are "locally" isomorphic using the method of variation of parameters (Theorems 1 and $1^{\prime}$ ). A patching argument using the $p$ adic Mittag-Leffler Theorem then shows they are "globally" isomorphic as well (Theorem 2). These results are all stated in terms of systems of first order linear differential operators. In $\S 4$ we consider the case of a single $k$ th order linear operator and obtain an explicit formula for the index. This is applied in $\S 5$ to

Received by the editors November 15, 1974.

AMS (MOS) subject classifications (1970). Primary 12B40, 34A30. Theorem.

Key words and phrases. $p$-adic numbers, index, regular singularity, Mittag-Leffler

( $\left.{ }^{1}\right)$ Research supported by an NSF Graduate Fellowship while the author was a student at Princeton University. 
obtain information on certain differential operators which were first studied by Dwork [5].

I am indebted to B. Dwork for many helpful discussions of these and related questions.

2. Definitions and preliminaries. We will be concerned with systems of operators of the form $D-A$, where $A=\left(a_{i j}(x)\right)$ is a $k \times k$ matrix with $a_{i j}(x) \in$ $\mathrm{C}_{p}(x), i, j=1,2, \ldots, k$, and $D=d / d x$ is applied to column vectors term by term. Suppose $V$ and $W$ are linear spaces of column vectors with meromorphic entries and that $D-A$ maps $V$ into $W$. If the kernel and cokernel of $D-A$ are finite dimensional, the index of $D-A$, denoted $\chi(D-A ; V, W)$, is defined by

$$
\chi(D-A ; V, W)=\operatorname{dim} \operatorname{ker}(D-A)-\operatorname{dim} \operatorname{coker}(D-A) .
$$

For disks of (additive) radius $r$ about a point $a \in \mathbf{C}_{p}$ we use the notation

$$
D(a, r-)=\left\{x \in \mathrm{C}_{p} \mid \operatorname{ord}(x-a)>r\right\}, \quad D(a, r+)=\left\{x \in \mathrm{C}_{p} \mid \operatorname{ord}(x-a) \geqslant r\right\}
$$

and for complements of sets we use the superscript $c$, e.g.,

$$
D(a, r+)^{c}=\left\{x \in \mathbf{C}_{p} \mid \operatorname{ord}(x-a)<r\right\} .
$$

For an open annulus of inner radius $r$ and outer radius $s$ about a point $a \in \mathrm{C}_{p}$, we use the notation

$$
U_{a}(s, r)=\left\{x \in \mathrm{C}_{p} \mid s<\operatorname{ord}(x-a)<r\right\} .
$$

For $n$ a nonnegative integer, let $\mathbf{C}_{p}^{n}[x]$ denote the vector space of all polynomials of degree $\leqslant n$, and let $C_{p}^{n}\left[(x-a)^{-1}\right]$ denote the vector space of all polynomials of degree $\leqslant n$ in $(x-a)^{-1}$.

Let $F(a ; r)$ be the set of all functions holomorphic on $D(a, r+)^{c}$ which vanish at $\infty$, i.e.,

$$
F(a ; r)=\left\{\xi \in(x-a)^{-1} C_{p}\left[\left[(x-a)^{-1}\right]\right] \mid \xi \text { converges on } D(a ; r+)^{c}\right\} \text {. }
$$

Let $F_{n}(a ; r)$ be the set of all functions holomorphic on $D(a, r+)^{c}$ except possibly for poles of order $n$ at $\infty$, i.e.,

$$
F_{n}(a ; r)=\left\{\xi_{1}+\xi_{2} \mid \xi_{1} \in F(a ; r), \xi_{2} \in \mathbf{C}_{p}^{n}[x]\right\} .
$$

Let $P(x)$ be a common denominator of the $a_{i j}(x)$, i.e., a polynomial such that the matrix $P(x) \cdot A$ has polynomial entries. Set

$$
N=\max _{i, j=1,2, \ldots, k}\left\{\operatorname{deg}\left(P \cdot a_{i j}\right)-1, \operatorname{deg} P-2,0\right\} .
$$

Then $D-A$ maps $F(a ; r)^{k}$ into $\left(F_{N}(a ; \dot{r}) / P(x)\right)^{k}$. If we put 


$$
G_{a}=\left(\frac{1}{x-a} \mathrm{C}_{p}\left[\frac{1}{x-a}\right]\right)^{k} \text { and } G_{a}^{n}=\frac{1}{P(x)}\left(\frac{1}{x-a} \mathrm{C}_{p}\left[\frac{1}{x-a}\right]+\mathrm{C}_{p}^{n}[x]\right)^{k}
$$

then $D-A$ maps $G_{a}$ into $G_{a}^{N}$.

For any formal Laurent series $\xi=\Sigma_{j=-\infty}^{\infty} c_{j}(x-a)^{j}$ about a point $a \in \mathrm{C}_{p}$ let

$$
\begin{aligned}
& \xi^{+}=\sum_{j=0}^{\infty} c_{j}(x-a)^{j} \in \mathrm{C}_{p}[[x-a]], \\
& \xi^{-}=\sum_{j=-\infty}^{-1} c_{j}(x-a)^{j} \in \frac{1}{x-a} \mathrm{C}_{p}\left[\left[\frac{1}{x-a}\right]\right]
\end{aligned}
$$

These definitions are extended to column vectors term by term.

We will make frequent use of the following result:

LEMma 1 (Dwork [3]). Consider the annulus $U_{a}(s, r), s<r<\infty$. Let $z$ be an element of $U_{a}(s, r)$, and let $v_{1}, v_{2}, \ldots, v_{n}$ be elements of $\mathbf{C}_{p}$, no two of which differ by a rational integer. For $i=1,2, \ldots, n$ let $\xi_{i}$ be a germ at $z$ of $(x-a)^{v_{i}}$ and let $\eta$ be a germ at $z$ of $\log (x-a)$. Let $M\left(U_{a}(s, r)\right)$ be the field of meromorphic functions on $U_{a}(s, r)$ and let $M_{z}$ be the field of germs of functions meromorphic at $z$. Then $M\left(U_{a}(s, r)\right)$ has a natural imbedding in $M_{z}$ and in this sense the elements $\left\{\xi_{i} \eta^{j}\right\} \quad\left(i=1, \ldots, n, j \in \mathbf{Z}_{\geqslant 0}\right)$ are linearly independent over $M\left(U_{a}(s, r)\right)$.

3. Local theory. A point $a \in \mathrm{C}_{p}$ is said to be a regular singular point of $D-A$ if at $a$ there exists a fundamental solution matrix of the form

$$
Y(x)=Z(x) B(x)(x-a)^{C},
$$

where $Z$ is a matrix whose coefficients lie in $C_{p}[[x-a]]$ and at least one entry has nonzero constant term, $B$ is a matrix whose coefficients are in $C_{p}[\log (x-a)]$, and $C$ is a diagonal matrix. The diagonal entries of $C$ are called the exponents of $D-A$ relative to $a$. We say that $D-A$ is non-Liouville at $a$ if every exponent $c$ relative to $a$ satisfies the condition $\operatorname{ord}(n-c) /|n|=o(1)$ as $n \rightarrow+\infty$ and as $n \rightarrow-\infty$ in Z (cf. Clark [2]). Here, "I |" denotes real absolute value. Note that in our terminology, regular singular points include ordinary points as a special case.

REMARK. If $D-A$ is obtained from a single $k$ th order differential operator $l$ by the usual process (see $\S 5$ ), and if the coefficients of $l$ satisfy the classical Fuchs' criterion at $a \in \mathrm{C}_{p}$, then $a$ is a regular singular point of $D-A$.

LEMMA 2. Suppose that $a \in \mathrm{C}_{p}$ is a regular singular point of $D-A$ and that $D-A$ is non-Liouville at $a$. Let $D\left(a, r^{-}\right)$be a disk about a on which the polynomial $P(x)$ defined in $\S 2$ has no zeros other than possibly $a$ and on which the matrix $Z(x)$ of (3.1) is holomorphic. Then every solution of the inhomogeneous equation 


$$
(D-A) \xi=\eta,
$$

with $\eta$ a $k \times 1$ column vector holomorphic on an annulus $U_{a}(r, s)(r<s)$, is a column vector whose entries are of the form

$$
\sum_{i=0}^{n} \mu_{i}(x) \log ^{i}(x-a)+\sum_{\text {finite }} v_{j}(x)(x-a)^{c_{j}} \quad(\text { polynomial in } \log (x-a))
$$

where the $c_{j}$ are certain of the exponents of $D-A$, all nonintegral and no two differing by an integer, and the $\mu_{i}$ and $\nu_{j}$ are holomorphic on $U_{a}(r, s)$. Furthermore, the powers of $\log (x-a)$ occurring in (3.3) are bounded by a constant which depends only on $D-A$.

PRoof. Every solution $\xi$ of (3.2) is of the form

$$
\xi=Y \int Y^{-1} \eta d x
$$

for some choice of indefinite integral, where $Y$ is the fundamental solution matrix (3.1). We examine first $\int Y^{-1} \eta d x$. By our hypothesis on $Y$, a typical entry $\tau$ in the column vector $Y^{-1} \eta$ has the form

$$
\tau=(x-a)^{-c} \sum_{j=0}^{n} \tau_{j}(x) \log ^{j}(x-a)
$$

where $c$ is one of the exponents and where the $\tau_{j}$ are holomorphic on $U_{a}(r, s)$. Such an entry can be integrated by parts, the point being that the uniform part of the resulting function is also holomorphic on $U_{a}(r, s)$ by the non-Liouville hypothesis. With this knowledge of $\int Y^{-1} \eta d x$, equation (3.4) and the hypothesis on $Y$ imply that $\xi$ has the required form. The final statement now follows from (3.4). Q.E.D.

REMARK. If in (3.2) $\eta$ is holomorphic on $D\left(a, r^{-}\right)$except for poles at $a$, then $\xi$ has entries of the form (3.3), where now the $\mu_{i}$ and $\nu_{j}$ are holomorphic on $D\left(a, r^{-}\right)$except for poles at $a$. This is easily checked by noting that none of the operations used in determining $\xi$ (equation (3.4)) will introduce an essential singularity.

The principal result of the local theory is

THEOREM 1. Suppose that $D-A$ is non-Liouville at the regular singular point $a \in \mathbf{C}_{p}$. Assume that the matrix $Z$ of (3.1) is holomorphic on a disk $D\left(a, r_{0}^{-}\right)$and that $P(x)$ has no zeros on $D\left(a, r_{0}^{-}\right)$except possibly at $a$. Then for $r>r_{0}$

and

$$
\operatorname{dim}\left(F_{N}(a ; r) / P(x)\right)^{k} /(D-A) F(a ; r)^{k}<\infty
$$




$$
G_{a}^{N} /(D-A) G_{a} \stackrel{1}{\longrightarrow} \frac{1}{P(x)} F_{N}(a ; r)^{k} /(D-A) F(a ; r)^{k}
$$

where the isomorphism is induced by the natural imbedding of $G_{a}^{N}$ into $F_{N}(a ; r)^{k} / P(x)$ ( $N$ is defined by (2.1)).

Proof. We may assume without loss of generality that $a=0$. We first prove the injectivity of (3.5).

We need to show that

$$
G_{0}^{N} \cap(D-A) F(0 ; r)^{k}=(D-A) G_{0} .
$$

Suppose there exists $\xi \in F(0 ; r)^{k}$ such that $(D-A) \xi=\eta \in G_{0}^{N}$. Then for some choice of indefinite integral we have $\xi=Y \int Y^{-1} \eta d x$. Lemma 2 and the remark following it show that the components of $\xi$ lie in $x^{-1} C_{p}\left[x^{-1}\right]+C_{p}[[x]]$, since $\xi$ is uniform. But $\xi \in F(0 ; r)^{k} \subset x^{-1} C_{p}\left[\left[x^{-1}\right]\right]^{k}$. Hence the components of $\xi$ lie in $x^{-1} \mathrm{C}_{p}\left[x^{-1}\right]$, i.e., $\xi \in G_{0}$.

For the surjectivity, let $\eta \in P^{-1} F_{N}(0 ; r)^{k}$ and set $\xi=Y \int Y^{-1} \eta d x$. Then

$$
(D-A) \xi=\eta
$$

and from Lemma 2 we know that

$$
\xi=\xi_{0}+\sum_{i=1}^{n} \xi_{i} \log ^{i} x+\sum_{i=n+1}^{m} \xi_{i} x^{c_{i}} \quad \text { (polynomial in } \log x \text { ) }
$$

where the $\xi_{i}, i=1, \ldots, m$, are column vectors holomorphic on $U_{0}\left(r_{0}, r\right)$ and the $c_{i}$ are all nonintegral, no two of them differing by an integer. Recall also that $n$ has a bound which depends only on $D-A$. We claim that the $\xi_{i}, i=$ $1,2, \ldots, n$, are holomorphic on $D\left(0, r_{0}^{-}\right)$except possibly for poles at 0 . We show this by descending induction on $i$. Equations (3.6) and (3.7) and Lemma 1 imply that

$$
(D-A) \xi_{n}=0 .
$$

Thus $\xi_{n}$ is a linear combination of column vectors of $Y$. We already know that $\xi_{n}$ is holomorphic on $U_{0}\left(r_{0}, r\right)$, hence it has no nonuniform terms. So by the hypothesis on $Y, \xi_{n}$ satisfies our claim. Suppose that $\xi_{i}$ satisfies the claim for some $i, 2 \leqslant i \leqslant n$. Then (3.6) and (3.7) and Lemma 1 show that

$$
(D-A) \xi_{i-1}=-\xi_{i} / x,
$$

so for some choice of indefinite integral $\xi_{i-1}=Y \int Y^{-1}\left(-x^{-1} \xi_{i}\right) d x$. Using the hypothesis on $Y$, the induction hypothesis, the remark following Lemma 2 , and the fact that $\xi_{i-1}$ is holomorphic on the annulus $U_{0}\left(r_{0}, r\right)$ (which means that $\xi_{i-1}$ contains no nonuniform terms), we now see that $\xi_{i-1}$ satisfies the claim. 
In particular, $\xi_{1}$ satisfies the claim, hence lies in $\left(x^{-1} \mathbf{C}_{p}\left[x^{-1}\right]+\mathbf{C}_{p}[[x]]\right)^{k}$. But the orders of the poles of $\xi_{1}$ are bounded by a number which depends only on $D-A$ and not on $\eta$. This is true because $\xi_{1}$ is determined recursively from (3.8) and (3.9) and because $n$ has a bound which depends only on $D-A$. Thus there exists a positive integer $J$ depending only on $D-A$ such that $\xi_{1} \in$ $\left(x^{-1} \mathbf{C}_{p}^{J}\left[x^{-1}\right]+\mathbf{C}_{p}[[x]]\right)$.

Equations (3.6) and (3.7) and Lemma 1 give

$$
\eta-(D-A) \xi_{0}^{-}=(D-A) \xi_{0}^{+}+\xi_{1} / x,
$$

with $\xi_{0}^{-} \in F(0, r)^{k}$. We have

$$
\eta-(D-A) \xi_{0}^{-} \in P^{-1} F_{N}(0 ; r)^{k} \subset P^{-1}\left(x^{-1} \mathbf{C}_{p}\left[\left[x^{-1}\right]\right]+\mathbf{C}_{p}^{N}[x]\right)^{k},
$$

but

$$
(D-A) \xi_{0}^{+}+x^{-1} \xi_{i} \in P^{-1}\left(x^{-1} \mathrm{C}_{p}^{J+1}\left[x^{-1}\right]+\mathrm{C}_{p}[[x]]\right)^{k} .
$$

Thus (3.10) shows that

$$
\eta-(D-A) \xi_{0}^{-} \in P^{-1}\left(x^{-1} \mathrm{C}_{p}^{J+1}\left[x^{-1}\right]+\mathrm{C}_{p}^{N}[x]\right),
$$

which implies $\eta-(D-A) \xi_{0}^{-} \in G_{0}^{N}$. This proves surjectivity and also that $\left(P^{-1} F_{N}(0 ; r)\right)^{k} /(D-A) F(0 ; r)^{k}$ is finite dimensional. Q.E.D.

The behavior of the cokernel of $D-A$ at $\infty$ is similar. We let $F(\infty ; r)$ be the space of all functions holomorphic on a disk about the origin of radius $r$, i.e.,

$$
F(\infty ; r)=\left\{\xi \in C_{p}[[x]] \mid \xi \text { converges on } D\left(0, r^{-}\right)\right\} \text {. }
$$

Then $D-A$ maps $F(\infty ; r)^{k}$ into $P(x)^{-1} F(\infty ; r)^{k}$ and maps $\mathrm{C}_{p}[x]^{k}$ into $P(x)^{-1} \mathrm{C}_{p}[x]^{k}$.

We say $\infty$ is a regular singular point of $D-A$ if $D-. A$ has a fundamental solution matrix there of the form

$$
Y(x)=Z(x) B(x)(1 / x)^{C},
$$

where $Z$ is a matrix with entries in $C_{p}\left[\left[x^{-1}\right]\right], B$ is a matrix whose entries are polynomials in $\log \left(x^{-1}\right)$, and $C$ is a diagonal matrix whose diagonal entries are called the exponents relative to $\infty$ (they are determined mod $Z$ ).

We have analogues of Lemma 2 and Theorem 1:

LEMMA 2'. Suppose that $\infty$ is a regular singular point of $D-A$ and that $D-A$ is non-Liouville at $\infty$. Let $r$ be such that the matrix $Z$ of (3.11) is holomorphic on $D\left(0, r^{+}\right)^{c}$ and such that $P(x)$ has no zeros on $D\left(0, r^{+}\right)^{c}$. Then every solution of the inhomogeneous equation

$$
(D-A) \xi=\eta,
$$


with $\eta$ a $k \times 1$ column vector holomorphic on $U_{0}(s, r)(s<r)$, is a column vector whose entries have the form

$$
\left.\sum_{i=0}^{n} \mu_{i}(x) \log ^{i}(1 / x)+\sum_{\text {finite }} v_{j}(x)(1 / x)^{c_{j}} \quad \text { (polynomial in } \log (1 / x)\right),
$$

where the $c_{j}$ are certain of the exponents of $D-A$, all nonintegral and no two differing by an integer, and the $\mu_{i}$ and $v_{j}$ are holomorphic on $U_{0}(s, r)$. Furthermore, the powers of $\log (1 / x)$ occurring in (3.13) are bounded by a constant which depends only on $D-A$.

PROof. Identical to the proof of Lemma 2 (or make the change of variables $x \rightarrow 1 / x$ and apply Lemma 2). Q.E.D.

REMARK. As before, if in (3.12) $\eta$ is holomorphic on $D\left(0, r^{+}\right)^{c}$ except for poles at $\infty$, then $\xi$ has entries of the form (3.13), where now the $\mu_{i}$ and $\nu_{j}$ are holomorphic on $D\left(0, r^{+}\right)^{c}$ except for poles at $\infty$.

THEOREM 1'. Suppose that $\infty$ is a non-Liouville, regular singular point of $D-A$. Let $r_{0}$ be such that the matrix $Z$ cf. (3.11) is holomorphic on $D\left(0, r_{0}^{+}\right)^{c}$ and such that $P(x)$ has no zeros on $D\left(0, r_{0}^{+}\right)^{c}$. Then for $r<r_{0}$,

$$
\operatorname{dim}(F(\infty ; r) / P(x))^{k} /(D-A) F(\infty ; r)^{k}<\infty
$$

and

$$
\left(\mathrm{C}_{p}[x] / P(x)\right)^{k} /(D-A) \mathrm{C}_{p}[x]^{k} \simeq(F(\infty ; r) / P(x))^{k} /(D-A) F(\infty ; r)^{k},
$$

where the isomorphism is induced by the natural imbedding of $\mathrm{C}_{p}[x] / P(x)$ into $F(\infty ; r) / P(x)$.

Proof. Identical to the proof of Theorem 1. Q.E.D.

4. Global theory. The global theory is now entirely straightforward. As in the local case, we will see that under appropriate hypotheses the holomorphic cokernel of $D-A$ is isomorphic to the cokernel of $D-A$ restricted to certain rational functions. The same will be true for the kernel of $D-A$, and hence for the index, also.

Let $\left\{a_{1}, a_{2}, \ldots, a_{n}\right\}$ be a set of points in $\mathrm{C}_{p}$, fixed for the remainder of this section, and let $r_{\infty}, r_{1}, r_{2}, \ldots, r_{n}$ be real numbers. The global object we will be concerned with is the space $F\left(r_{\infty}, r_{1}, r_{2}, \ldots, r_{n}\right)$ of functions holomorphic in the region

$$
S=D\left(0, r_{\infty}^{-}\right)-\bigcup_{i=1}^{n} D\left(a_{i}, r_{i}^{+}\right) .
$$

From now on, we always assume that the disks $D\left(a_{i}, r_{i}^{+}\right), i=1,2, \ldots, n$, are 
contained in $D\left(0, r_{\infty}^{-}\right)$and are pairwise disjoint. Under this hypothesis the $p$-adic Mittag-Leffler Theorem holds (see, for example, Robba [8]):

$$
F\left(r_{\infty}, r_{1}, r_{2}, \ldots, r_{n}\right)=F\left(\infty ; r_{\infty}\right) \oplus \sum_{i=1}^{n} \bigoplus F\left(a_{i} ; r_{i}\right) .
$$

When there is no danger of confusion, we denote $F\left(r_{\infty}, r_{1}, r_{2}, \ldots, r_{n}\right)$ simply by $F$. Then $D-A$ maps $F^{k}$ into $P^{-1} F^{k}$.

From now on, we assume also that $a_{1}, \ldots, a_{n}, \infty$ are regular singular points of $D-A$. For each $a_{i}, i=1,2, \ldots, n$, let $D\left(a_{i}, s_{i}^{-}\right)$be the uncircumferenced disk on which the matrix $Z$ of (3.1) converges, and let $D\left(0, s_{\infty}^{+}\right)^{c}$ be the uncircumferenced disk about $\infty$ on which the matrix $Z$ of (3.11) converges. We suppose that $P(x)$ has no zeros on $D\left(0, s_{\infty}^{+}\right)^{c} \cup\left(\bigcup_{i=1}^{n} D\left(a_{i}, s_{i}^{-}\right)\right)$except possibly $a_{1}, \ldots, a_{n}$. Let $E$ denote the set of rational functions with poles only at $a_{1}, a_{2}, \ldots, a_{n}, \infty$, i.e.,

$$
E=\mathrm{C}_{p}\left[x,\left\{\left(x-a_{i}\right)^{-1}\right\}_{i=1}^{n}\right] .
$$

The operator $D-A$ maps $E^{k}$ into $P^{-1} E^{k}$.

LEMMA 3. If $r_{\infty}<s_{\infty}$ and $r_{i}>s_{i}, i=1,2, \ldots, n$, then $\operatorname{ker}\left(D-A, F^{k}\right) \subset E^{k}$.

Proof. Let $\xi \in \operatorname{ker}\left(D-A, F^{k}\right)$. Then $\xi$ is holomorphic on $U_{a_{i}}\left(s_{i}, r_{i}\right)$ for each $i$ (a nontrivial annulus since $r_{i}>s_{i}$ ) and coincides there with a linear combination of solutions of $D-A$ on $D\left(a_{i}, s_{i}\right)$. The function $\xi$ contains no nonuniform terms because it is holomorphic on an annulus, hence can be analytically continued to all of $D\left(a_{i}, s_{i}^{-}\right)$except for possible poles at $a_{i}$. This process works at $\infty$ as well, thus $\xi$ can be analytically continued to a function holomorphic everywhere except for possible poles at $a_{1}, \ldots, a_{n}, \infty$. Such a function must be rational, and the conclusion of the lemma follows. Q.E.D.

The main result of the global theory is now easy using the Mittag-Leffler Theorem and our local results:

THEOREM 2. If $r_{\infty}<s_{\infty}, r_{i}>s_{i}, i=1,2, \ldots, n$, and if $D-A$ is nonLiouville at $a_{1}, \ldots, a_{n}, \infty$, then the natural imbedding of $E$ into $F$ induces an isomorphism

$$
P^{-1} E^{k} /(D-A) E^{k} \stackrel{\sim}{\rightarrow} P^{-1} F^{k} /(D-A) F^{k} .
$$

Furthermore, $\operatorname{dim} P^{-1} F^{k} /(D-A) F^{k}<\infty$.

Proof. For the injectivity, let $\eta \in P^{-1} E^{k}$ and suppose there exists $\xi \in F^{k}$ with $(D-A) \xi=\eta$. We may write $\eta=\eta_{\infty}+\sum_{i=1}^{n} \eta_{i}$ with $\eta_{\infty} \in P^{-1} \mathbf{C}_{p}[x]^{k}$, $\eta_{i} \in P^{-1}\left(\left(x-a_{i}\right)^{-1} \mathbf{C}_{p}\left[\left(x-a_{i}\right)^{-1}\right]\right)^{k}, i=1,2, \ldots, n$, and by Mittag-Leffler $\xi=\xi_{\infty}+\Sigma_{i=1}^{n} \xi_{i}$ with $\xi_{\infty} \in F\left(\infty ; r_{\infty}\right)^{k}, \xi_{i} \in F\left(a_{i} ; r_{i}\right)^{k}$. For every $i$ we must have 
$(D-A) \xi_{i}=\eta_{i}+q_{i}$ where $q_{i} \in P^{-1} \mathrm{C}_{p}^{N}[x]^{k}$. But then Theorem 1 implies that there exists $\tilde{\xi} \in\left(x-a_{i}\right)^{-1} \mathbf{C}_{p}\left[\left(x-a_{i}\right)^{-1}\right]^{k}$ such that $(D-A) \widetilde{\xi}_{i}=\eta_{i}+q_{i}$. We now have

$$
(D-A) \xi_{\infty}=\eta_{\infty}-\sum_{i=1}^{n} q_{i},
$$

and by Theorem 1' there exists $\widetilde{\xi}_{\infty} \in \mathbf{C}_{p}[x]^{k}$ such that

$$
(D-A) \widetilde{\xi}_{\infty}=\eta_{\infty}-\sum_{i=1}^{n} q_{i} .
$$

Hence putting $\widetilde{\xi}=\widetilde{\xi}_{\infty}+\sum_{i=1}^{n} \widetilde{\xi}_{i}$, we have $\widetilde{\xi} \in E^{k}$ and $(D-A) \widetilde{\xi}=\eta$, so the map is injective.

For surjectivity, take $\eta \in P^{-1} F^{k}$. Multiplying (4.1) by $P^{-1}$ we see that we may write $\eta=\eta_{\infty}+\Sigma_{i=1}^{n} \eta_{i}$ with $\eta_{\infty} \in P^{-1} F\left(\infty ; r_{\infty}\right)^{k}, \eta_{i} \in P^{-1} F\left(a_{i} ; r_{i}\right)^{k}, i=$ $1,2, \ldots, n$. For each $i$ there exist, by Theorem 1 , functions

$$
\xi_{i} \in P^{-1}\left(\left(x-a_{i}\right)^{-1} \mathrm{C}_{p}\left[\left(x-a_{i}\right)^{-1}\right]\right)^{k}, q_{i} \in P^{-1} \mathrm{C}_{p}^{N}[x]^{k},
$$

and $\mu_{i} \in F\left(a_{i} ; r_{i}\right)^{k}$ such that

$$
\eta_{i}=\xi_{i}+q_{i}+(D-A) \mu_{i} .
$$

By Theorem 1', there exists $\xi_{\infty} \in P^{-1} \mathbf{C}_{p}[x]^{k}, \mu_{\infty} \in F\left(\infty ; r_{\infty}\right)^{k}$ such that

$$
\eta_{\infty}=\xi_{\infty}+(D-A) \mu_{\infty} \text {. }
$$

Adding (4.3) and (4.2) over $i=1,2, \ldots, n$ gives

$$
\eta=\xi_{\infty}+\sum_{i=1}^{n}\left(\xi_{i}+q_{i}\right)+(D-A)\left(\mu_{\infty}+\sum_{i=1}^{n} \mu_{i}\right) \text {. }
$$

Since $\xi_{\infty}+\Sigma_{i=1}^{n}\left(\xi_{i}+q_{i}\right) \in P^{-1} E^{k}$ and $\mu_{\infty}+\Sigma_{i=1}^{n} \mu_{i} \in F^{k}$, we have surjectivity.

The last statement follows because the $\xi_{i}, q_{i}, \xi_{\infty}$ can be chosen to lie in a finite dimensional space (Theorems 1 and 1'). Q.E.D.

Corollary. Under the hypotheses of Theorem 2,D-A has index and

$$
\chi\left(D-A ; F^{k}, F^{k} / P\right)=\chi\left(D-A ; E^{k}, E^{k} / P\right) .
$$

PROof. Immediate from Theorem 2 and Lemma 3. Q.E.D.

Although we do not know an explicit formula for $\chi\left(D-A ; E^{k}, P^{-1} E^{k}\right)$ it can usually be calculated without great difficulty.

REMARK. Theorem 2 shows that we can choose a basis for $P^{-1} F^{k} /(D-A) F^{k}$ which consists of rational functions and that furthermore this basis may be chosen to be independent of $r_{\infty}, r_{1}, r_{2}, \ldots, r_{n}$, as long as $r_{\infty}<s_{\infty}, r_{i}>s_{i}, i=1,2$, $\ldots, n$. 
5. Example. In this section we obtain a formula for the index of a single $k$ th order linear differential operator with polynomial coefficients. Let $l$ be the differential operator

$$
l=P_{k}(x) D^{k}+P_{k-1}(x) D^{k-1}+\cdots+P_{1}(x) D+P_{0}(x)
$$

where $P_{0}, P_{1}, \ldots, P_{k} \in \mathrm{C}_{p}[x]$. We consider also the equivalent system of operators $D-A$, where

$$
A=\left[\begin{array}{c|c}
0 & I_{k-1} \\
\hline-P_{0} / P_{k} & -P_{1} / P_{k} \cdots-P_{k-1} / P_{k}
\end{array}\right]
$$

We may take $P=P_{k}$ in $\S 4$, and when the hypotheses of Theorem 2 are satisfied we have

$$
\begin{aligned}
& \frac{1}{P_{k}} E^{k} /(D-A) E^{k} \stackrel{\sim}{\rightarrow} \frac{1}{P_{k}} F^{k} /(D-A) F^{k} \\
& { }_{E^{k} / P_{k}(D-A) E^{k} \stackrel{\sim}{\longrightarrow} F^{k} / P_{k}(D-A) F^{k}}
\end{aligned}
$$

where the vertical arrows are isomorphisms induced by multiplication by $\boldsymbol{P}_{\boldsymbol{k}}$ and the horizontal arrows (induced by the imbedding $E \rightarrow F$ ) are isomorphisms by Theorem 2. Thus, $P_{k} \cdot(D-A)$ is an endomorphism of $E^{k}$ and of $F^{k}$ and

$$
\chi\left(P_{k} \cdot(D-A) ; F^{k}, F^{k}\right)=\chi\left(P_{k} \cdot(D-A) ; E^{k}, E^{k}\right) .
$$

The operator $l$ is an endomorphism of $E$ and of $F$. Let $u: F \rightarrow F^{k}$ be defined by $u(\xi)=\left(\xi, \xi^{\prime}, \ldots, \xi^{(k-1)}\right)$ and let $v: F \rightarrow F^{k}$ be defined by $v(\xi)=$ $(0,0, \ldots, 0, \xi)$. Then the diagram

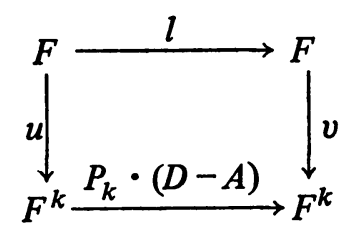

commutes and induces an isomorphism between the kernel of $l$ and the kernel of $P_{k} \cdot(D-A)$, and between the cokernel of $l$ and the cokernel of $P_{k} \cdot(D-A)$; the same is true with $F$ replaced by $E$ and we get

$$
\begin{gathered}
\chi(l ; F, F)=\chi\left(P_{k} \cdot(D-A) ; F^{k}, F^{k}\right) \text { and } \\
\chi(l ; E, E)=\chi\left(P_{k} \cdot(D-A) ; E^{k}, E^{k}\right) .
\end{gathered}
$$

Hence under the hypotheses of Theorem 2, we have by (5.1) 


$$
\chi(l ; F, F)=\chi(l ; E, E) .
$$

REMARK. We note for future use that, under the hypotheses of Theorem 2, we can choose a basis for $F / l F$ which consists of rational functions and that this basis may be chosen independent of $r_{\infty}, r_{1}, \ldots, r_{n}$ as long as $r_{\infty}<s_{\infty}, r_{1}>s_{i}$, $i=1,2, \ldots, n$.

We now compute the right side of (5.2). This formula is probably well known, but we do not know a suitable reference, so we will give the details. It is first necessary to introduce some notation.

For $c \in \mathbf{C}_{p}, f \in \mathbf{C}_{p}[x]$, let ord $c$ fenote the order of the zero of $f(x)$ at $c$. For the differential operator $l$, let

$$
R_{c}=\max _{i=0,1, \ldots, k}\left\{i-\operatorname{ord}_{c} P_{i}(x)\right\} .
$$

As vector space over $\mathrm{C}_{p}$, a basis for $E$ is given by $\left\{x^{j}\right\}_{j=0}^{\infty} \cup\left(\bigcup_{i=1}^{n}\left\{\left(x-a_{i}\right)^{-j}\right\}_{j=1}^{\infty}\right)$. For $\bar{N}=\left(N_{\infty}, N_{1}, N_{2}, \ldots, N_{n}\right) \in\left(\mathrm{Z}_{\geq 0}\right)^{n+1}$, let $E_{\bar{N}}$ be the subspace of $E$ spanned by $\left\{x^{j}\right\}_{j=1}^{N_{\infty}} \cup\left(\bigcup_{i=1}^{n}\left\{\left(x-a_{i}\right)^{-j}\right\}_{j=1}^{N_{i}}\right)$. If $e \in \mathbf{Z}_{\geq 0}$, let $\bar{e}=(e, e, \ldots, e)$ $\in\left(\mathrm{Z}_{\geqslant 0}\right)^{n+1}$. If $\bar{M}=\left(M_{\infty}, M_{1}, M_{2}, \ldots, M_{n}\right)$, we say that $\bar{N} \geqslant \bar{M}$ if $N_{\infty} \geqslant M_{\infty}$, $N_{i} \geqslant M_{i}, i=1,2, \ldots, n$. We say $\bar{N}>\bar{M}$ if $N_{\infty}>M_{\infty}, N_{i}>M_{i}, i=1,2, \ldots, n$. Define a positive integer $N^{\prime}$ by

$$
N^{\prime}=\max _{i=0,1, \ldots, k}\left\{\operatorname{deg} P_{i}-i\right\} .
$$

Proposition 1. The index of $l$ on $E$ is given by

$$
\chi(l ; E, E)=-\left(N^{\prime}+\sum_{i=1}^{n} R_{a_{i}}\right) .
$$

Proof. Let $\bar{R}=\left(N^{\prime}, R_{a_{1}}, R_{a_{2}}, \ldots, R_{a_{n}}\right)$. Then $l$ maps $E_{\bar{N}}$ into $E_{\bar{N}+\bar{R}}$ whenever $\bar{N}+\bar{R}>\overline{0}$. Let $\operatorname{ker}\left(l, E_{\bar{N}}\right)=\left\{\xi \in E_{\bar{N}} \mid l \xi=0\right\}$. We have an exact sequence

$$
0 \rightarrow \operatorname{ker}\left(l, E_{\bar{N}}\right) \rightarrow E_{\bar{N}} \stackrel{l}{\rightarrow} E_{\bar{N}+\bar{R}} \rightarrow E_{\bar{N}+\bar{R}} / l E_{\bar{N}} \rightarrow 0 ;
$$

thus

$$
\begin{aligned}
\operatorname{dim} \operatorname{ker}\left(l, E_{\bar{N}}\right)-\operatorname{dim} E_{\bar{N}+\bar{R}} / l E_{\bar{N}} & =\operatorname{dim} E_{\bar{N}}-\operatorname{dim}_{\bar{N}+\bar{R}} \\
& =\left(N^{\prime}+\sum_{i=1}^{n} R_{a_{i}}\right) .
\end{aligned}
$$

Choose $\bar{N}$ so large that $\operatorname{ker}(l, E) \subset \operatorname{ker}\left(l, E_{\bar{N}}\right)$ (hence they are equal) and such that each component of $\bar{N}$ is large enough to insure that for $\bar{M} \geqslant \bar{N}, \operatorname{deg} l\left(x^{M_{\infty}}\right)$ $=M_{\infty}+N^{\prime}$ and that as a polynomial in $\left(x-a_{i}\right)^{-1}, \operatorname{deg} l\left(\left(x-a_{i}\right)^{-M_{i}}\right)=M_{i}+R_{a_{i}}$. Let $H$ be a complementary subspace of $l E_{\bar{N}}$ in $E_{\bar{N}+\bar{R}}$, i.e., 


$$
E_{\bar{N}+\bar{R}}=l E_{\bar{N}} \oplus H .
$$

We claim that for any $\bar{M} \geqslant \bar{N}$,

$$
E_{\bar{M}+\bar{R}}=l E_{\bar{M}} \oplus H .
$$

Note first that by our choice of $\bar{N}$,

$$
\begin{aligned}
E_{\bar{M}+\bar{R}}= & l\left(\mathrm{C}_{p} \cdot x^{M_{\infty}}\right) \oplus l\left(\mathrm{C}_{p} \cdot x^{M_{\infty}-1}\right) \oplus \cdots \oplus l\left(\mathrm{C}_{p} \cdot x^{N_{\infty}+1}\right) \\
& \oplus \sum_{i=1}^{n} l\left(\mathrm{C}_{p} \cdot \frac{1}{\left(x-a_{i}\right)^{M_{i}}}\right) \oplus l\left(\mathrm{C}_{p} \cdot \frac{1}{\left(x-a_{i}\right)^{M_{i}-1}}\right) \\
& \oplus \cdots \oplus l\left(\mathrm{C}_{p} \cdot \frac{1}{\left(x-a_{i}\right)^{N_{i}+1}}\right) \oplus E_{\bar{N}+\bar{R}} .
\end{aligned}
$$

Equation (5.5) now follows from (5.4) and (5.6). Equation (5.5) implies that $E=l E \oplus H$. The proposition now follows from (5.3). Q.E.D.

Note that the only hypothesis in Proposition 1 is that $l$ have polynomial coefficients. From (5.2) we have

COROLlary 1. Under the hypotheses of Theorem 2,

$$
\chi(l ; F, F)=-\left(N^{\prime}+\sum_{i=1}^{n} R_{a_{i}}\right) .
$$

Using the hypotheses of Theorem 2 we can simplify the right side of (5.7). Since we are assuming $\infty$ is a regular singular point of $l$ we must have (by Fuchs' criterion) $\operatorname{deg} P_{k} \geqslant \operatorname{deg} P_{j}+(k-j)$ for $j=0,1, \ldots, k$. Hence

$$
N^{\prime}=\operatorname{deg} P_{k}-k \text {, }
$$

and since each $a_{i}$ is a regular singularity, we get (by Fuchs' criterion)

$$
\operatorname{ord}_{a_{i}} P_{j} \geqslant \operatorname{ord}_{a_{i}} P_{k}-(k-j)
$$

for $j=0,1, \ldots, k$. Hence

$$
R_{a_{i}}=k-\operatorname{ord}_{a_{i}} P_{k} .
$$

Adding (5.8) and (5.9) over $i=1,2, \ldots, n$ gives

$$
N^{\prime}+\sum_{i=1}^{n} R_{a_{i}}=(n-1) k+\operatorname{deg} P_{k}-\sum_{i=1}^{n} \operatorname{ord}_{a_{i}} P_{k} .
$$

But recall that part of the hypothesis of Theorem 2 is that all the zeros of $P_{k}$, except possibly $a_{1}, a_{2}, \ldots, a_{n}$, lie in the region $S$. So if we let $v\left(P_{k}, S\right)$ be the number of zeros of $P_{k}$ on $S$, then 


$$
V\left(P_{k}, S\right)=\operatorname{deg} P_{k}-\sum_{i=1}^{n} \operatorname{ord}_{a_{i}} P_{k} .
$$

Thus, from Corollary 1 follows

COROLLARY 2. Under the hypotheses of Theorem $2, \chi(l ; F, F)=k(1-n)-$ $v\left(P_{k}, S\right)$.

REMARK 1. The restriction on the zeros of $P_{k}$ can be weakened slightly. Consider the operator $P l$, where $P$ is any rational function with no poles on $S$ and $l$ satisfies the hypotheses of Theorem 2. Multiplication by $P$ is stable on $F$ and has index, so

$$
\chi(P l ; F, F)=\chi(P ; F, F)+\chi(l ; F, F) .
$$

But $\chi(P ; F, F)=-v(P, S)$, hence

$$
\chi(P l ; F, F)=k(1-n)-v\left(P P_{k} ; F, F\right) .
$$

REMARK 2. For differential operators with coefficients in $\mathrm{Q}(X)$, the proofs of all our results are valid over $\mathbf{C}$ as well as over $\mathbf{C}_{p}$ (when the coefficients are imbedded in $\mathbf{C}(x)$ ). Hence Corollary 2 agrees with the result in the classical (i.e., complex) case. In fact, over $\mathbf{C}$ this result is valid under very weak hypotheses (see Malgrange [6]). Specifically, it is not necessary to assume that the excluded disks contain only regular singular points of the differential operator. However, a different method of proof is needed in the case of irregular singularities because (5.2) is not always true in this case (over $\mathbf{C}$ or over $\mathbf{C}_{p}$ ). This is easily seen by considering the differential operator satisfied by the exponential function: $D-1$. This same example shows that Corollary 2 is false in the case of irregular singularities in $\mathbf{C}_{p}$, at least without additional hypotheses.

6. Application. We discuss some of the questions that motivated our previous work. From now on we assume $p$ is an odd prime. Let $g(x) \in \mathrm{C}_{p}[x]$ be defined by

$$
g(x)=\sum_{j=0}^{p-1 / 2}\left(\frac{(1 / 2)_{j}}{j !}\right)^{2} x^{j}
$$

where for each nonnegative $j$ and each $c \in \mathbf{C}_{p}$,

$$
(c)_{j}=\sum_{s=0}^{j-1}(c+s) \text { for } j>0, \quad(c)_{0}=1 .
$$

For each pair of positive real numbers $b_{1}, b_{2}$, let $L\left(b_{1}, b_{2}\right)$ be the set of all functions holomorphic and bounded in the region

$$
\left\{x \in \mathrm{C}_{p} \mid \text { ord } g(x)<b_{1}, \max \left(\text { ord } x, \text { ord } x^{-1}, \text { ord }(x-1)\right)<b_{2}\right\},
$$


let $L=\operatorname{dir} \lim _{b_{1}, b_{2}>0} L\left(b_{1}, b_{2}\right)$ with the obvious inclusions.

Let

$$
\tilde{l}_{1}=D^{2}+\frac{(1-2 x)}{x(1-x)} D-\frac{1}{4 x(1-x)}
$$

The differential operator $\tilde{l}_{1}$ is a classical hypergeometric operator (see Poole [7]). It has Wronskian $w=(x(1-x))^{-1}$ and its only singularities are regular singularities at $0,1, \infty$. Let $u_{1}, u_{2}$ be two linearly independent formal solutions of $\widetilde{l}_{1}$ at some point $x_{0} \in \mathbf{C}_{p}$. For $k \in \mathbf{Z}_{+}$, the $k$ th symmetric power of $\widetilde{l}_{1}$, denoted $\widetilde{l}_{k}$, is defined to be the (unique) linear differential operator with leading coefficient 1 whose space of formal solutions at $x_{0}$ is the $\mathbf{C}_{p}$-vector space with basis $\left\{u_{1}^{j} u_{2}^{k-j}\right\}_{j=0}^{k}$. Let $l_{k}$ be the (unique) linear differential operator with leading coefficient 1 whose space of formal solutions at $x_{0}$ is the $\mathrm{C}_{p}$-vector space with basis $\left\{w^{-k} u_{1}^{j} u_{2}^{k-j}\right\}_{j=0}^{k}$. It is the $k$ th symmetric power of

$$
l_{1}=D^{2}+\left(w^{\prime} / w\right) D+\left(\left(w^{\prime} / w\right)^{2}+7 w / 4\right) .
$$

We will compute the dimensional of $L / l_{k} L$. For the number theoretic significance of this calculation, we refer the reader to [1] and [5].

Consider the operator $l_{k}$ on the space of holomorphic functions $F$ with $\left\{a_{1}, a_{2}, \ldots, a_{n}\right\}=\{0,1\} \cup\{x \mid g(x)=0\}$. Then it is well known that the hypotheses of Theorem 2 are satisfied with $s_{\infty}=s_{1}=s_{2}=\cdots=s_{n}=0$ (see Dwork [4]), and in this case

$$
\chi\left(l_{k} ; F, F\right)=-(k+1)((p+1) / 2) .
$$

Since $l_{k}$ has trivial kernel on $F$,

$$
\operatorname{dim} F / l_{k} F=(k+1)((p+1) / 2) .
$$

Let $c_{1}, c_{2}, \ldots, c_{p-1 / 2}$ be the zeros of $g(x)$. From the remark following (5.2), we conclude that there is a subset

$$
H \subset \mathrm{C}_{p}\left[x, x^{-1},(x-1)^{-1},\left\{\left(x-c_{j}\right)^{-1}\right\}_{j=1}^{p-1 / 2}\right]
$$

such that $H$ is a basis for $F / l_{k} F$ which is independent of $r_{\infty}, r_{1}, r_{2}, \ldots, r_{n}$. Thus

$$
\operatorname{dim} L\left(b_{1}, b_{2}\right) / l_{k} L\left(b_{1}-\epsilon_{1}, b_{2}-\epsilon_{2}\right)=(k+1)((p+1) / 2)
$$

when $b_{-1}, b_{2}, \epsilon_{1}, \epsilon_{2}, b_{1}-\epsilon_{1}, b_{2}-\epsilon_{2}$ are all positive, hence

$$
\operatorname{dim} L / l_{k} L=(k+1)((p+1) / 2) \text {. }
$$

\section{REFERENCES}

1. A. Adolphson, Thesis, Princeton University, 1974.

2. D. Clark, $A$ note on the p-adic convergence of solutions of linear differential equations, Proc. Amer. Math. Soc. 17 (1966), 262-269. MR 32 \#350. 
3. B. M. Dwork, On p-adic analysis, Some Recent Advances in the Basic Sciences, Vol. 2 (Proc. Annual Sci. Conf., Belfer Grad. School Sci., Yeshiva Univ., New York, 1965-1966), Belfer Graduate School of Science, Yeshiva Univ., New York, 1969, pp. 129-154. MR 41 \#5687.

4. - p-adic cycles, Inst. Hautes Études Sci. Publ. Math. No. 37 (1969), 27-115. MR 45 \#3415.

5. - On Hecke polynomials, Invent. Math. 12 (1971), 249-256. MR 46 \#1735.

6. B. Malgrange, Sur les points singuliers des équations différentielles, Seminaire Goulaouic-Schwarz, 1971-1972.

7. E. G. C. Poole, Introduction to the theory of linear differential equations, Dover, New York, 1960. MR 22 \#2746.

8. P. Robba, Fonctions analytiques sur les corps values ultra-me'triques complets, Thèse, Univ. Paris VII, 1972.

DEPARTMENT OF MATHEMATICS, UNIVERSITY OF WASHINGTON, SEATTLE, WASHINGTON 98195 